to prevent the direct light from the lamps reaching the candidate's eyes, but these are made with matt black non-reflecting surfaces. In this way uniformity of illumination is secured on the card and avoidance of failing illumination through dirt or deterioration of a reflecting surface. The card itself should be matt white (not varnished) and the testing room moderately illuminated in such a way that no glaring lights or bright objects come in the candidate's field of vision.

These objects can all be secured at very small cost, and they represent the minimum which should be aimed at in fairness to all candidates. But we strongly endorse the dictum of the Council as to the desirability of all such examinations being held at properly equipped centres. Every ophthalmic surgeon has during the last four-and-a-half years had experience of the difficulties of examining men and making decisions under adverse circumstances and with equipment quite inadequate for the purpose. We know of a case where an ophthalmic surgeon worked for eighteen months, and examined hundreds of men, in a draughty corridor with confusing cross lights, and amidst the constant noise of passing trollies. Dare we hope that when ultimately the dream of a Ministry of Health is realized, one of its early tasks will be to institute a properly equipped centre for the full medical examination of all candidates for the public services?

We heartily congratulate the Council of British Ophthalmologists on its first published report, and hope that its recommendations will receive general acceptance.

\title{
ABSTRACTS
}

\section{I.-METASTATIC OPHTHALMITIS}

(I) Pichler (Klagenfurt). - Sudden paresis of the pupil as the first sign of a metastatic ophthalmia. (Plötzliche Pupillenlähmung als erstes Zeichen einer metastatischen Ophthalmie.) Klin. Monatsbl. f. Augenheilk., June, I9I 5, p. 682.

(1) Pichler noted in one case of pneumonia, in one of pyaemia, and in one of meningitis and pneumonia, that the pupil of one eye suddenly became dilated and did not contract to. light. Either at the same time, or within two or three days, metastatic ophthalmia was found to be present. All the cases were fatal.

H. M. TRAQUAIR. 
(2) Szily, A. von.-Metastatic ophthalmia following war injuries. Atlas der Kriegsaugenheilkunde, Stuttgart, I9ı6.

(2) In this book Szily devotes a chapter to metastatic ophthalmia, following war injuries, and gives full clinical and pathological details of four cases. The condition is rare. Not a single instance has occurred at the 2nd London General Hospital during the four years of the war, although a very large number of surgical cases have been treated there. Szily's cases, therefore, are of interest.

CASE I.-G. J. J., aged 29. Wounded, August 15, 1914, by a rifle bullet which entered behind the right ear, penetrated the mouth, and destroyed the superior maxilla, not injuring the eyes either directly or indirectly. There was also a severe wound of the buttock. When seen by Szily, September 12, 1914, there was exophthalmos of the right eye with chemosis, haziness of the aqueous, iritis, and a grey fundus reflex. The left eye also was affected, but to a lesser degree. On September 21, 1914, panophthalmitis was fully established, and the patient died September 25, 1914. Pneumococci were recovered from the vitreous, and also from cultures.

Post-mortem examination.-Pneumonia, pleurisy, pericarditis, septic infarcts in the kidneys and spleen. The eyes were examined, and showed evidence of septic inflammation, with the presence of the pneumococcus in all the structures.

Three to four weeks elapsed between the dates of the infliction of the wound and the involvement of the eyes.

CASE II.-B. M., aged 32, wounded August 24, 1914, by shellcasing in the left side of the neck. Operated on November 3, 1914, for a carotid aneurism. Subsequently he had attacks of raised temperature, and the wound was reopened January 18, 1915. In February, 1915, an attempt was made to remove the metal which was wedged in the cervical vertebrae, but unsuccessfully. March 12, 1915, left eye shows signs of inflammation. Exophthalmos, chemosis; later on, iritis and panophthalmitis. Enucleation performed March 24, 1915, streptococci being recovered from the vitreous. The right eye was not involved. Pathological examination showed the eye to be a myopic one; complete detachment of the retina with inflammation of the whole of the retina, choroid, ciliary body, etc. Between the date of the wound and the onset of the panophthalmitis a period of nearly eight months elapsed, but there was only one month between the last operation and the inflammatory onset in the eye.

CASE III.-A. R., aged 24, wounded June 24, 1916, by a piece of shell casing in the right thigh. June 26, 1916, wound attacked by gas gangrene and amputation resorted to. Subsequently the patient developed a general pyaemia. July 7, 1916, the sight of the left eye became involved and the case was seen by Szily who diagnosed 
metastatic ophthalmia and removed the eye July 15, 1916. Staphylococci were recovered from the patient's blood and also from the vitreous. Patient died November 15, 1916.

Post-mortem examination-Purulent metastatic lobular pneumonia of the left lung was found, also septic endocarditis. The eyes showed general septic inflammation of all the tissues and Gram-positive cocci were found in the retina. Two weeks only elapsed between the date of the wound and the onset of panophthalmitis.

CASE IV.-Qu. St., aged 25, wounded June 29, 1916, by an artillery shot in the lower extremity. June 30, 1916, two wounds (a) just below the knee in the soft parts, $(b)$ compound fracture of the tibia just above the ankle joint. Temperature raised, headache, etc. Leg was put up in a plaster of Paris bandage. July 23, 1916, inflammation of left eye. Ciliary injection, aqueous hazy, iritis. July 25, 1916, L. E. removed on account of panophthalmitis. Typical chain cocci found in the vitreous. July 27, 1916, patient died.

Post-mortem examination.-Cellulitis of the leg, with septic thrombosis of the femoral vein. Metastatic abscesses in the neck, rectus muscle, epididymis, etc., endocarditis.

Szily from his examination of these cases comes to the conclusion that in the first three cases the eyes were infected through the retinal vessels and the last through the ciliary vessels of the iris and ciliary body. From the pathological standpoint the cases are dealt with fully.

ARTHUR W. ORMOND.

\section{(3) Coriat and Boulat.-A case of bilateral metastatic ophthal- mitis consecutive to war wounds. (Un cas d'ophtalmie métastatique bilatérale, consécutive à une blessure de guerre.) Arch. d'Ophtal., May-June, I9I 8.}

(3) Metastatic ophthalmitis attacking both eyes is sufficiently uncommon to justify the record of individual examples. The case under the care of Coriat and Boulat is reported with full clinical details, including the temperature chart, and will repay study by those interested. The chief features of the case are as follows :

The patient, a soldier, aged 29, was wounded by shell fragments on September 26, 1916, and admitted to hospital on September 29. $\mathrm{He}$ had a deeply penetrating wound in the left sacro-iliac region, with a fragment of shell embedded close to the sacro-iliac symphysis, and a second but superficial wound of the right thigh. The latter wound healed readily; the former was suppurating, but not severely and the patient was able to walk about comfortably. On December 18, the fragment of shell was extracted through a large incision, with removal of some bone. This was followed next day by severe pain in the left leg, along the sciatic nerve, and a 
sharp rise of temperature. The wound on the fifth day was healthy and there was very little pus. On December 26, the man complained of severe pains in several joints, especially on movement. On January 12, slight conjunctival congestion of the right eye was noted. On January 13, there were dilatation and immobility of the left pupil, and dimness of vision; the general condition of the patient had become much worse, although a free escape of pus from a cavity at the bottom of the wound had occurred.

From this date signs of acute ophthalmitis rapidly developed in both eyes, and ultimately perforation of both corneae occurred. The patient died on January 26. Unfortunately no autopsy was made.

In this case, as in nearly all similar observations hitherto published, the ophthalmitis may be considered as the manifestation of an infection of long duration. Chenet, in his interesting thesis on this subject, has shown that metastatic ophthalmitis usually occurs in the course of chronic. suppuration, in which septicaemia is slowly established. In the 46 cases collected by him, the date of appearance of ocular signs varied from one to forty days. In the case now reported the first signs of ocular involvement were noted $3 \frac{1}{2}$ months after the wound, and at a time when the multiple joint lesions testified to the spread of pathogenic germs in the circulation.

A noteworthy point in this case is the rapid increase in severity of the symptoms from the time at which the eyes become involved. Before the eyes were attacked the patient seemed to strive with more or less success against infection; as soon as ocular infection occurred, resistance weakened and the disease made rapid progress. It would seem, as Chenet has said, that "ophthalmitis is a true sign of the defeat of the human organism, which has been maintaining for a long period a struggle against invasion by pyogenic microbes, derived from the infected wound."

The prognosis in metastatic ophthalmitis is grave and especially so in cases which are bilateral. Chenet's thesis contains twentytwo examples of bilateral ophthalmitis all of which terminated fatally; death occurred from the first to the fourteenth day after the onset of the ocular lesions. Coriat and Boulat's patient died fourteen days after the first ocular manifestations.

J. B. LAWFORD. 


\section{II.-OPERATIONS}

\section{Keratoplasty}

(I) Magitot, A. (Paris)-A critical study of certain biological properties of the corneal tissue and of human keratoplasty. (Etude critique sur certaines propriétés biologiques du tissue cornéen et sur la kératoplastie humaine.) $A n n$. d'Ocul., September and October, I9ı6.

(1) Magitot has a suggestive article dealing with the bearing of the biological properties of the corneal tissue upon the success or failure of the operation of keratoplasty. It should be read in the original, since it is difficult to do justice to its contents even in a lengthened abstract, such as follows.

\section{Anatomy}

From the present point of view, the cornea presents two main anatomical elements, namely, the epithelium and the parenchyma. In discussing the peculiarities of these two parts, Magitot brings out no particularly new facts. He points out, however, that elastic fibres, which are derived from the fixed corneal cells, are present only towards the periphery of the cornea, in front of Descemet's membrane. Again, he insists that the cornea is particularly tolerant in certain directions, as, for example, towards some inert foreign bodies. Following the idea of Pellier de Quengsy (1789), many have covered leucomatous corneae with prothetic appliances. The tolerance of these contrivances seldom exceeds a few weeks, although there are exceptions to that statement. Thus, Salzer, employing small fixative hooks of gold, succeeded in keeping in place a crystal cornea for two and a half years in one case and for eight months in another. The same author, after superficial keratectomy, grafted a piece of horse's cornea, killed by immersion in formol. Without actually assimilating the foreign body, the cornea supported it so well that Salzer wished to compare the result with that obtained from living grafts.

\section{The Regeneration of Corneal Tissue}

In order to understand the different problems presented by keratoplasty, it is necessary to know how the corneal tissue repairs itself.

We must distinguish between the repair of $(a)$ an aseptic wound, and $(b)$ a wound caused by an inflammatory lesion.

(a) Regeneration of the corneal tissues after the infliction of an aseptic wound takes place as follows.-The lips of the divided 
parenchyma show changes, as rarefaction of the lamellae, with the formation of lacunae, and swelling of the fixed cells, with fragmentation (Retterer). A wound of the parenchyma, then, leads to decay of the anatomical elements. The epithelium, on the contrary, manifests great activity. First, there is a sinking in of the elements along the edges of the wound (Ranvier). which represents an active movement, the result of which is the formation of new cells. It is not comparable with the sand gliding along the sides of a declivity. On the contrary, it results from an active movement, which starting at the limbus, is transmitted to the cells at the edges of the wound. After the second hour, in fact, one sees mitotic figures in the basal cells of the epithelium, a process that commences at the limbus.

The karyokinetic wave, so to speak, causes the marginal cells to fall into the wound. In this way they come to carpet the solution in continuity, more and more cells being pushed into the gap by successive waves originating from the limbus. The work proceeds so rapidly that Löwenstein has estimated that a loss of substance as considerable as 12 sq. $\mathrm{mm}$. is covered in sixty hours. The cellular activity is accompanied by a dilatation of the vessels of the limbus, as well as by the appearance of small capillaries in the parts adjacent to the wound. The presence of these vessels appears to be indispensable, for if the sclero-corneal limbus be cauterized, the repair of a traumatism is much slackened. The single layer of flat epithelial cells augments in thickness, and as new elements are born from hour to hour, there is soon produced a supplementary layer and then another. The stratification resulting from this process has no tendency to cease until, by the accumulation of successive layers, the bottom of the wound is approximately brought to the normal low-water mark. In consequence of the proximity of the sclero-corneal vessels, the work proceeds more quickly towards the periphery than the centre of the cornea.

The wound is by these means covered with epithelium, but for several weeks there remains a depression, or facette, visible by focal illumination. In the course of five to six months, this disappears, little by little, by regeneration of the parenchyma. The origin of the fibroblasts (or keratoblasts) was believed by Ranvier to be from the fixed cells of the parenchyma, but Retterer is of the view that they originate mainly from the epithelium, and that the fixed cells play but a subordinate part. The conclusions reached by Retterer are based upon important observations made by Bonnefon and Lacoste, Salzer, and Magitot himself. In point of fact, the keratoblasts are to be found from the first in large numbers immediately beneath the epithelium and in contact with it. In many spots they may be found in the interval between the epithelial cells, or in the space between two detached epithelial layers. Magitot admits the epithelial origin of the keratoblasts (at least, until the contrary is 
proved), and this he does apparently for two reasons.-1. At first the epithelial cells are the only ones to show exuberance and vitality, while the parenchyma surrounding the wound presents merely signs of degeneration. 2. The normal fixed cells, a little farther from the wound, remain inert, and are not capable of aiding in the regenerative process until much later.

(b) The regeneration of a septic wound of the cornea, of which Magitot takes a pneumococcal ulcer as the type, opens up a different set of conditions for consideration. The corneal lesion is deprived of all epithelium. In the acute phase the microbic elements have their zone of activity, not in the bottom of the wound, but mainly at its edges.

The epithelial cells, then, have fallen before they have any chance of gliding into the depths of the wound. The usual phenomena of inflammation simultaneously make their appearance. The fixed cells of the parenchyma become transformed into macrophages. When the infection becomes extinct, the epithelium, little by little, recovers its activity, and gains the wound. The corneal lamellae, deeply dissociated by the leucocytic infiltration, have lost their rectilinear stratification, and have become thickened and wavy, while the gaps which separate them are filled with degenerate cytological elements. Above all, they have lost their primordial quality, transparency, and a leucoma is the result. As the outcome of several factors, the leucoma may improve.-First, the cellular débris lying between the lamellae may be slowly resorbed. Secondly, the newly-formed capillaries may disappear. Thirdly, there is a regeneration of transparent tissue, and this is less pronounced the deeper the ulcerative process, and vice versa. It is probable that the histological repair of corneal infections is not identical for each infection, while there are certainly differences as regards infections and burns, as by acid or by lime. The truth of this assertion is attested by the fact that a graft is better accepted by the latter than by the former. Magitot points out that: (1) the excision of a morsel of a leucoma leaving cicatricial tissue around, results only in the formation of a new opaque tissue; (2) if the leucoma be completely excised, transparent regeneration is possible, as in Malgaigne's case ; and (3) if a leucomatous disc be transplanted into healthy tissue, it is found at the end of several months that the opacity of the graft has notably diminished.

\section{The Grafts}

A piece of healthy cornea taken from an animal and transplanted into the sound cornea of the other eye, retains its transparency and rapidly adheres, provided the operation has been carried out aseptically. The adhesion of the graft, which occurs in several hours, is chiefly due to the epithelium of the grafted eye. The 
tissue of the parenchyma of the graft survives, but it appears that the fixed cells die. These cells are replaced immediately by others which result from the proliferation of the fixed cells of the grafted eye. At the end of several months, then, the graft is represented by its epithelium and by most of its tissue proper, in which may be , found elements of new formation, derived from the grafted eye. If the graft be transplanted into the cornea of an animal of the same species as that from which it was taken, at first coalescence and transparency are observed, but the last-named is perhaps a little less perfect, although it tends to improve. The conditions are different when a corneal graft is transplanted into the eye of another species. Successful transplantation as between guinea-pig and rabbit has been carried out, but both are rodents belonging to the mammalia. On the other hand, as between the hen and the rabbit, the graft is merely tolerated and not assimilated. At the end of several weeks, the epithelium of the graft is absorbed by that of the host, and the tissue proper is completely destroyed and replaced by that of the host. With the foregoing data in mind, we may make the following classification.-(a) Autoplasty, when the graft has been taken from the same individual; (b) homoplasty, when the graft has been derived from an individual of the same species; and (c) heteroplasty, when the graft has been taken from an individual of a different species. Many heteroplasties have been performed upon man, and all the domestic animals have been drawn upon for the purpose. Yet there has never been a lasting success. Magitot hints, however, that there may be a chance of success if anthropoids are employed. As regards man, autoplasty gives 95 per cent. and homoplasty about 60 per cent. of successes. The conditions are difterent in man and animals : in human beings one has to deal with an abnormal tissue, whereas in animals the cornea is normal.

Provided the wound is aseptic; the corneal tissue is capable of undergoing spontaneous transparent regeneration, as may be witnessed in certain traumatisms of the human cornea. It may be equally seen at the end of several months, after we have removed a flap of transparent tissue, but it is quite otherwise when dealing with a piece of leucomatous tissue. As pointed out before, in order to obtain a good result, we must remove entirely the cicatricial tissue under strict aseptic conditions. This view is borne out by experiments made by Magitot upon cases of pterygium where the cornea is invaded.

It is unfortunate that all leucomata are not capable of being equally benefited by the operation of keratoplasty. Pathological anatomy shows how different these cicatrices are. The reason is two-fold : first, the cause; and, secondly, the extent of the corneal disturbances. Of the numerous affections entailing the production of blemishes, infections occupy a predominating place. Those due 
to the pneumococcus or Petit's bacillus give rise to ulcerations which, although having a variable extent, are circumscribed. The regions not actually invaded by the microbes retain, when inflammation has subsided, not only their transparency; but show little thickening of the parenchyma. It is otherwise in interstitial keratitis. The corneal lamellae remain dissociated and infiltrated with cells of new formation and lymphocytes. At the level of the leucoma the cornea is doubled or tripled in thickness. From what we know of the treponema, it is probable that some of these microorganisms remain in the tissue, which explains the fact that attempts to transplant sound tissue have provoked renewed inflammation. Another affection in which the results are disappointing is pemphigus. On the other hand, keratoplasty may be expected to give its full eftect in the cicatrices left by ulcers and superficial burns and in pterygium.

Another important condition is the extent and particularly the depth of the cicatrix. If it does not extend to the membrane of Descemet, the result of keratoplasty is likely to be good as regards transparency. If the contrary is the case, total keratoplasty alone remains, and that operation is still in its infancy and bristles with difficulties. It is clearly important, therefore, to be able to gauge the depth of the scar. 'There is, however, only one certain way of doing this, viz., by operation.

\section{The Elements of Success and of Failure}

Magitot next discusses the elements of success and failure in keratoplasty. In the first rank he places the question of soil (terrain), and then he mentions the several points of the extent and the depth of the leucoma and of its origin. The choice of graft is also important. The best chances lie in autoplasty. If the periphery of the leucomatous cornea is healthy, the flap should be taken from that eye, and the best place is the zone covered by the upper eyelid. Under other circumstances, it may be necessary to take the graft from the other eye, but, in principle, if that eye is quite sound, it is better not to touch it, and to get the graft from another person, although the chances of success are diminished by doing so. The graft should not be thick. The reason for this lies in the fact that the tissue proper of the cornea swells in taking up water. It is impossible to prevent this from taking place, for despite all care, we cannot hinder the tears from coming into contact with it. Even during the dissection, the graft has already doubled in thickness. It follows that, however quickly it is placed in position, the graft projects from the general plane of the cornea. It has no tendency to settle down until several days have passed, and meanwhile there is always the risk of displacement. In short, the piece of tissue transplanted should be slenderer than the portion of 
leucoma excised. Another reason why the graft should be thin is that the only parts of service are the epithelium and the lamellae lying immediately beneath. In order to avoid perforation of the anterior chamber, it is well to be provided with two or three special instruments. Magitot does not approve Hippel's trephine for cutting the flap, but prefers trephines after the Bowman type, having a diameter of $4 \mathrm{~mm}$. or $5 \mathrm{~mm}$. He detaches the graft by a special instrument, of which the blade is set at an angle of 45 degrees with the handle. The utmost care must be taken not to displace the graft while removing the speculum from the eye.

As to the causes of failure in keratoplasty, Magitot lays stress upon opening of the anterior chamber during the operation. Should this accident take place, the lips of the corneal parenchyma become swollen by imbibition of aqueous humour, which prevents coalescence of the graft and is only too likely to lead to detachment of the same. The most rigid asepsis is, of course, necessary. Asepsis of the conjunctival culs-de-sac is to be secured by employing repeated eye baths of serum, and, finally, by mechanically cleansing the conjunctiva with Locke's formula, ${ }^{*}$ without glucose, or in default of that liquid, with serum, employing a curved and flat canula for the purpose. In order to avoid injury, the graft should be touched as little as possible. As regards anaesthesia, Magitot uses a 2 per cent. solution of cocain, and does not exceed that strength. The eye that has been operated on is covered with a warm moist dressing and cotton wool. The binocular bandage is left alone for forty-eight hours, and the eye is then bathed with serum. On the third day the binocular is replaced by a monocular dressing. This is replaced by dark glasses on the sixth day. For the first four or five days the graft is slightly prominent, but this convexity disappears in about a fortnight.

\section{The Operation itself and its Accidents}

Magitot next enters into a minute description of $(a)$ partial keratoplasty, (b) transcorneal keratoplasty, and (c) total keratoplasty. It seems unnecessary to follow him into these descriptions, although a word may be said as to certain incidents that may attend the operations.

In partial keratoplasty the anterior chamber may be opened, usually during dissection of the leucoma. The wound may be trivial, in which case the aqueous will escape drop by drop, moistening the flap and determining its swelling by imbibition. Under these circumstances, as also when the aqueous escapes with a gush, the

\footnotetext{
*Locke's artificial serum contains : sodium chloride, $1 \mathrm{gm}$.; potassium chloride, $0.01 \mathrm{gm}$. ; calcium chloride, $0.02 \mathrm{gm}$. ; sodium bicarbonate, $0.02 \mathrm{gm}$.; glucose, $0.1 \mathrm{gm}$.; and water, 100 cc.-" The Extra Pharmacopoeia," Vol. II, p. 310, 1915.
} 
operation should be interrupted for a month. On the other hand, the surgeon, desirous of avoiding evacuation of the aqueous humour, may cleave the cornea in such a way as to remove but an insignificant thickness of the leucoma. If the mistake is observed at the beginning, there is nothing to prevent the point of the needle from being withdrawn and introduced at a neighbouring spot. On the contrary, if the fault is not noticed until after part of the flap has been dissected, the best plan is to liberate the flap in three-fourths of its circumference, to seize it with small forceps, to turn it over, and to continue the slitting (clivage) in a deeper plane. By exerting too great pressure with the fixation forceps, the graft may be torn during dissection, and of this, supposing the graft to heal, traces will be visible for months. When the flap has been placed in the cavity prepared for its reception, it may become displaced by movements of the eyelid. It should be replaced at once. In order to avoid this accident, the speculum should be removed with care, and the patient be advised to close the eye gently, as in sleep.

\section{Conclusion}

The last nine pages of Magitot's communication are devoted to a discussion of the whole question. A healthy cornea is capable of transparent repair, provided the wound has not become infected. Even a large excision of corneal tissue from man may be repaired without leucoma. In about a year, the resulting facette is almost unrecognizable, while the irregular astigmatism has markedly diminished.' The amount of repair naturally varies with the extent and the depth of the loss of substance. Magitot noted that it took seven to nine months to render imperceptible a trephine aperture in the thickness of the periphery of the cornea, $4 \mathrm{~mm}$. in diameter and five- to six-tenths of a millimetre in thickness. The nervous sensibility of the affected area probably never returns quite to normal.

In the matter of pathological tissue, the difficulties are greater. Laboratory animals are but little susceptible to the microbic elements to which the human cornea is. so sensitive. The infections set up experimentally either exceed their end or else are extremely fugacious. Burns entail indelible scars in men, but in animals (when the eyes are not wholly destroyed), after a period of suppuration, a transparent repair of the parenchyma takes place. In man, the aseptic excision of a morsel of a leucoma provokes considerable vascularization and ends in the production of a more opaque cicatrix. To that rule there are, however, important exceptions. For example, certain scars resulting from superficial burns may become regenerated in a transparent manner, even if the trephine does not include the whole of the cicatricial tissue. The tissue, however, must not be too dense, and, above all, too profound. In a word, it seems that: 
regeneration is possible only if in the depths of the wound, the instrument has bitten into healthy parenchyma. The leucomata which result from ulcers can be ablated with advantage only if the whole of the cicatricial tissue be removed, both as regards extent and depth. Clinical experience shows us, in fact, that in this respect there is a great difference between this kind of cicatrix and that which results from burns.

In considering the grafts, we must take account of three different questions: the operative technique, the material of the graft, and the soil. Partial keratoplasty is regarded as uniting the minimum of inconveniences, while transcorneal keratoplasty is looked upon as exposing the patient to all the dangers of a penetrating wound of the eyeball. That is the current view. But by perfecting the technique of the last-named operation it has been found that it can be performed with a minimum of danger. The errors of appreciation as between the results of the two operations arise from the material transplanted and the nature of the soil. It is agreed that the best chances of success are given by the autoplastic plan, whereas with homoplasty the percentage of happy cases falls rapidly. In man, heteroplasty has so far furnished nothing beyond numerous failures. The fate of the graft is still a controversial point. Does the graft ultimately disappear, being replaced little by little by new tissue from the cornea, or is it able, on the contrary, to survive on its own account, merely borrowing from the surrounding tissue nutritive materials? In considering this question, we must first remember that the normal cornea is remarkably tolerant towards aseptic foreign bodies, and particularly towards organic foreign bodies. A piece of dead cornea, fixed by protoplasmic coagulation with the aid of formol, may be borne without reaction for several years (Salzer), although finally it is eliminated. The author himself believes that an autoplastic corneal graft is almost always accepted. The nature of the soil destined to receive the transplant is of the first importance. The leucomatous tissues of men are not alike among themselves, owing partly to their diverse causes and partly to their extent and depth. It is unfortunately true that in man an autoplastic graft plunged in the midst of a cicatrix following an ulcer does not retain its transparency beyond a few days. Burns occupy a place apart.

In conclusion, the author tells us that he has performed keratoplasty about forty times, almost always partial keratoplasty. The results have almost invariably been excellent in leucomata determined by burns, and almost invariably unfavourable when the cicatricial tissue has resulted from ulceration. In fact, the only two cases where a good result was obtained in the last-named class were in patients in whom transplantation was carried out after complete resection of the corneal scar. As matters stand, burns, pterygia 
(simple or cicatricial), and trachomatous leucomata may be benefited by the operation. It is possible that in the future this list may be considerably extended. Magitot lays stress upon the advisability of employing very thin grafts. The ideal would be the purely epidermic graft proposed by Bonnefon and Lacoste. As, however, a Thiersch's corneal graft is not easily to be obtained, we must in the meanwhile be content with one of minimum thickness. The last word has yet to be spoken on the subject of grafts.

S. S.

\section{(2) Walker, Sydney, junr. (Chicago). - Present status of corneal transplantation and some experimental data. Ophthal. Record, August, 1917.}

(2) Summarizing his study of the literature of corneal transplantation, Walker concludes that foreign body transplants (glass, celluloid, and so forth) and heteroplasty are bound to lead to failure, and that autoplasty and homoplasty ofter the greatest chance of success. By experimenting upon the eyes of dead dogs, Walker found it impossible in those animals to make corneal grafts with a conjunctival flap attached, as advocated by Lohlein. He accordingly decided upon a modified Thiersch graft, and applied the method to twelve living dogs. The eyes were treated for three weeks by the nightly instillation of protargol ( 3 per cent.) and by washing them out each morning with sublimate $(1: 4,000)$. Operation was not undertaken until a bacteriological examination showed that the conjunctival sac was free from virulent micro-organisms, particularly the streptococcus. Meanwhile the dogs were kept in fumigated cages.

The operation was performed as under.-The ocular conjunctiva was loosened up over the whole area extending from seven to four o'clock on the dial, so that it could be readily pulled over the entire cornea without much tension. After bleeding had been carefully stopped, the corneal flap was cut with a Graefe cataract knife,. and three or four fine silk ligatures were passed through its corners, for which purpose the author designed special forceps. Meanwhile the host's cornea was prepared in the way described above, and the flap; moistened with normal salt solution, was laid upon the prepared cornea. The sutures attached to the graft were inserted just deep enough to hold, and when all were in place they were pulled taut, so as to approximate but not to strangulate the tissue. The threads were cut off close, and the eye was washed with normal salt solution. The conjunctival flap was next brought over the entire cornea and sutured. In dogs, the third eyelid was sutured over the flap and the lids were bandaged. Forty-eight hours after operation the conjunctival flap was loosened, and any remaining corneal sutures were taken away. Dionin (5 per cent.) and weak sublimate irrigations 
were used. At this stage the corneal graft was clouded, and there was some injection, but the process tended to clear, and the final result was a leucoma discernible under strong light. After three or four months there was little to show how extensive the flap had been.

In three of the twelve experimental dogs, where the minimum amount of blood was allowed to remain when the conjunctival flap was brought over the cornea, the result appears to have been good. Four months after operation the eyes looked normal and the surface was absolutely smooth and regular.

Walker further operated upon three patients, whose cases are abstracted below :

(1) Female, 26 years, with dense leucomata covering central third of cornea in both eyes as the result of small-pox. Autoplasty upon one eye alone. The whole of the leucoma was not removed. On loosening the conjunctival flap on the second day, the graft was found to be attached only at its upper border, and was later brushed off by the upper eyelid.

(2) Male, aged 23 years, with slight opacity of the central region of the corneal surface. Homoplasty. At present time graft in place, and the slight cloudiness and organization appears to be gradually disappearing under atropin and dionin.

(3) Female, 19 years, slight opacity quarter cornea from phlyctenular ulcer. Autoplasty. The graft is at present in place, and "cloudliness and organization no more than expected at-this time."

Most of Walker's earlier failures were due to sepsis and illdeveloped technique where the anterior chamber was punctured. Later, opacities and organization were due mostly to too much blood in contact with the flap.

\section{Conclusions}

1. Heteroplasty and foreign body grafts offer very small chance of success, and should be discarded, according to the literature.

2. Autoplasty and homoplasty at the present time will terminate with fair results, depending on the technique of operation.

3. The modified Thiersch graft is a simple, satisfactory, and expedient manner of obtaining graft.

4. The conjunctival flap, as described, offers sufficient nutrition with the minimum cloudiness and organization, besides serving to hold the graft in position while union is taking place. results.

5 . With properly selected cases this operation will give fair S. S. 


\section{III.-INTERSTITIAL KERATITIS}

\section{Derby, George S. (Boston). - Interstitial keratitis, with special reference to the end-result. Ophthal. Record, November, 1917.}

Derby's communication is based upon 96 cases of interstitial keratitis, in most of which the inflammation had terminated at least two years previously. In 94 of the cases both eyes had been aftected. The total number of eyes examined therefore was 190. In 168 of 182 eyes, or 92.30 per cent., more or less opacity of the cornea could be made out under careful examination. In 171 of 186 eyes, or $92 \cdot 93$ per cent., vessels were found in the cornea after the pupil had been dilated and the parts examined with an ophthalmoscope and a strong convex lens. As bearing upon the view that these fine vessels seldom, if ever, disappear, as held by Fuchs, Hirschberg, Silex, Saemisch, Galezowski, and others, Derby mentions a case where they were found to be present 55 years after the original inflammation, and he recalls Baas's observation where, although absent clinically, they were found on histological examination.

Posterior synechiae were present in 62 of the eyes in Derby's series. His experience leads him to think that the involvement of the iris and ciliary body often bears little relationship to the severity of the corneal process. In 4 of the eyes included in Derby's series slight opacification of the lens was noted. In 2 eyes, vitreous opacities were found, but in 38 eyes it was impossible to make a satisfactory examination of the deeper structures of the eye. Of 148 eyes, 81 , or 54.73 per cent., showed lesions of the choroid and retina, usually in the form of disseminated lesions in the equatorial region. The author does not doubt that in some so-called negative cases lesions lay anterior to the equatorial region. The examination was made with care, with dilated pupil, and particular attention was paid to the periphery of the fundus. It so happened that the eyes were examined in two groups. In the first the percentage was $54^{\bullet} 3$, and in the second 55. Derby believes that in the majority of cases the fundus lesions appear independently of the corneal process, and usually antedate the latter.

Of 161 eyes examined under a cycloplegic and with the proper correction, Vision was $10 / 10$ in $32 ; 7 / 10$ in $17 ; 5 / 10$ in $18 ; 4 / 10$ in $13 ; 3 / 10$ in $25 ; 2 / 10$ in $14 ; 1 / 10$ in 17 ; and less than $1 / 10$ in 25. In another table Derby gives the vision of the better eye in 80 cases. From this it appears that vision was $10 / 10$ in 24 eyes; $7 / 10$ in 9 eyes; $5 / 10$ in 11 eyes; $4 / 10$ in 5 eyes; $3 / 10$ in 11 eyes; $2 / 10$ in 9 eyes; $1 / 10$ in 6 eyes; and less than $1 / 10$ in 
5 eyes. These figures show that 24 children would have the same chance educationally as the normal child; 25 more could study in the same classes as normal children, but would be handicapped in choosing an occupation; 20, or possibly 26 , could be educated in defective eyesight classes, or, failing that, would have to be referred to institutions for the blind, as would the remaining 5 .

The relation of interstitial keratitis to tuberculosis has never been definitely settled, and the views of authors differ markedly with regard to the point. Derby, after quoting some of these views, evidently thinks that while most cases are due to syphilis, some are due to tubercle or to other causes.

In respect of recurrences, Derby found positive evidence of recurrence in 14 of 37 cases, and in 3 more he thought that recurrence was probable.

Finally, Derby mentions a couple of cases where the treatment of hereditary syphilis in early life did not prevent the development of specific affections of the eye later. At the same time he expresses a strong conviction that such discouraging cases should not deter us from the energetic treatment of affected children. He closes with the following quotation from Igersheimer: "In the interest of the later career of patients with interstitial keratitis it is important to administer antisyphilitic treatment with the greatest energy, not only during the course of the ocular disease, but afterwards from time to time as long as the Wassermann reaction is positive."

S. S.

\section{CORRESPONDENCE}

\section{OPHTHALMIA NEONATORUM}

To the Editor, The BRitish Journal of Ophthalmology.

DEAR SIR,

In the current issue of the Journal there is a note on the establishment of a hospital for infants afflicted with ophthalmia neonatorum and their mothers by the Metropolitan Asylums Board; and ascribing the impetus that secured this new provision to the Report of the Departmental Committee on the Welfare of the Blind. So far as my information goes this is scarcely correct; rather the British Medical Association should have been credited with the successful action.

In June, 1917, the Council of the Metropolitan Branch of the Association appointed a deputation (Mrs. Scharlieb, Messrs. Treacher 